\title{
Panamá: la brecha digital en las noticias
}

\author{
Urribarri, Raisa \\ Centro Internacional de Estudios Políticos y Sociales (CIEPS) \\ Ciudad de Panamá, Panamá \\ ORCID:0000-0002-6466-125X
}

\section{Abstract}

We present the results of a documentary investigation on the coverage about the digital divide made by the Panamanian media during the first six months of the pandemic generated by COVID-19 (March-September). Based on the theoretical postulates of Jan van Dijk, the digital access gap is framed within the context of an unequal society. The coverage of this phenomenon is reviewed based on two of the main criteria that govern journalistic theory and practice, such as newsworthiness and selection of news sources. The analysis of 144 informative pieces reveals that the discontent expressed by social groups that had difficulties in accessing online education was the trigger for a profuse media coverage, but with little explanatory depth.

Keywords: Digital divide, COVID-19, Inequality, Media, Panama.

\section{Resumen}

En esta ponencia presentamos los resultados de una investigación documental sobre la cobertura que hicieron los medios de comunicación social de Panamá acerca de la brecha digital durante los seis primeros meses de la pandemia generada por la COVID-19 (marzoseptiembre). Con base en los postulados teóricos de Jan van Dijk, se enmarca la brecha digital de acceso dentro del contexto de una sociedad desigual. Se revisa la cobertura de este fenómeno por parte de los medios de comunicación de Panamá con base en dos de los principales criterios que rigen la teoría y la práctica periodística, como son la noticiabilidad y selección de las fuentes noticiosas. El análisis de 144 piezas informativas revela que el descontento expresado por los grupos sociales que tuvieron dificultades para acceder a la educación en línea fue el detonante de una cobertura mediática profusa, pero de poca profundidad explicativa.

Palabras claves: Brecha digital, COVID-19, Desigualdad, Medios de comunicación, Panamá. 


\section{INTRODUCCIÓN}

La crisis sanitaria originada por la COVID-19 evidenció que los extraordinarios recursos que provee Internet, y las innumerables ventajas que estos comportan, no son accesibles a todos por igual.

Para analizar la cobertura de la brecha digital por parte de los medios de comunicación de Panamá se partió de dos aproximaciones teóricas. Por un lado, la de Van Dijk (2020) relativa a la brecha digital, con la que se analiza la situación panameña. Por su parte, la cobertura de este fenómeno por parte de los medios de comunicación se hizo con base en las nociones de noticiabilidad y selección de las fuentes noticiosas propuestas por Wolf (1987) y Martini (2000).

Para el análisis se seleccionó un corpus de 144 piezas informativas difundidas en el periodo comprendido entre marzo y septiembre de 2020. Los resultados muestran que la brecha digital fue el detonante del descontento expresado por los grupos sociales que tuvieron dificultades para acceder a la educación en línea, un hecho que generó una cobertura mediática profusa, pero intermitente y de poca profundidad explicativa.

\section{MÉTODO}

La revisión de la cobertura mediática sobre la brecha digital se llevó a cabo en cuatro fases y solo tomó en cuenta los medios con presencia digital, pues la mayoría de los medios de Panamá cuentan con ella. En la primera fase, se hizo un seguimiento aleatorio de los temas asociados a la brecha digital que ocuparon la agenda informativa. En la segunda, mediante una búsqueda de cuatro términos o palabras clave, acotada en el período comprendido entre los meses de marzo y septiembre, se precisaron los medios que los abordaron, lo cual arrojó una colección de 140 piezas publicadas en cinco diarios de empresas editoriales diferentes (La Prensa, La Estrella-El Siglo, y Panamá América-Crítica); las plataformas de televisión y radio de los grupos TVN (TVN y TVN Radio) y Medcom (Telemetro y RPC Radio); y el medio nativo digital Capital Financiero. En la tercera fase, estas piezas se organizaron por tipo de medio (prensa, radioeléctricos y nativos digitales), tópico, género, autor, y fuentes consultadas. Finalmente, en la cuarta fase se analizó el contenido de las piezas, para detectar el tópico tratado y las fuentes consultadas para abordarlo.

\section{RESULTADOS}

En primer lugar, hay que destacar que el tópico que llevó la brecha digital a convertirse en noticia fue el educativo, el cual concentró el $84 \%$ de las piezas recolectadas en el periodo de estudio.

El mes que acumula la mayor cantidad de notas es agosto, con 44 piezas. De éstas, el $30 \%$ estuvo referido a un hecho de protesta que, bajo una inusual consigna "iLiberen la 
banda ancha!", fue convocado por los integrantes de la Coalición Nacional por la Defensa de la Educación Oficial en Panamá (AEVE Panamá 2020) para expresar su descontento por la imposibilidad de acceder a la educación en línea.

Sin duda este fue un hecho absolutamente inusual, que afectaba a un considerable número de personas, y con la potencialidad de generar conflictos. A pesar de estas características, y de las consecuencias que podrían generarse, el tema educativo, en general, prontamente dejó de ocupar espacios relevantes en la agenda informativa, con apenas 12 notas el siguiente mes.

Figura 1.
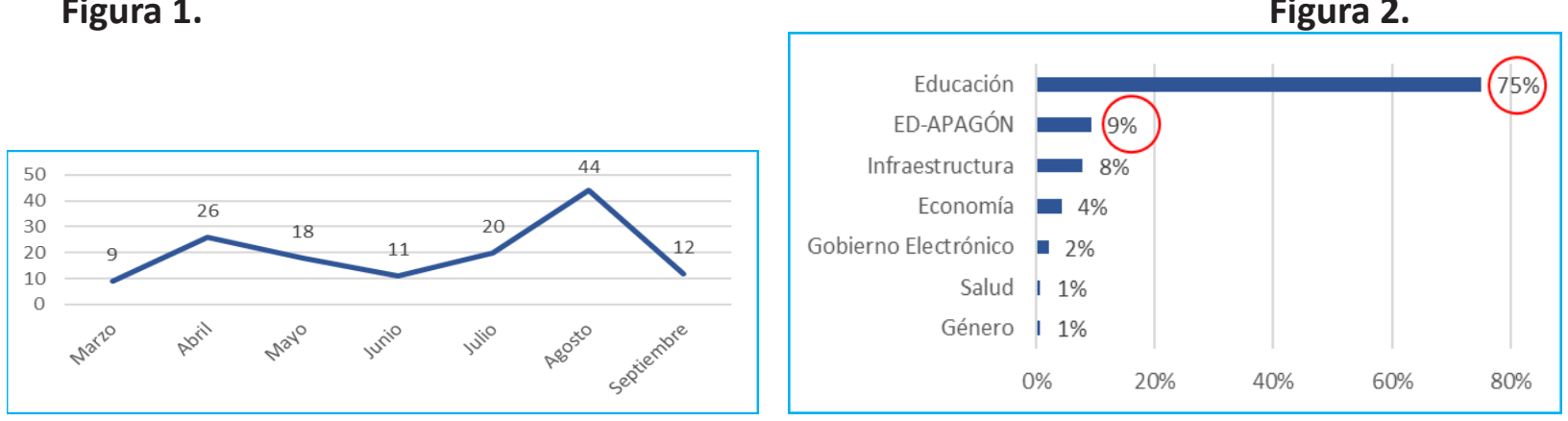

El análisis igualmente revela la preeminencia de la fuente única, preferentemente la oficial, proveniente de gacetillas o notas de prensa, cuestión que se advierte en el 39\% de las notas publicadas. Asimismo, la mayoría de las notas que dan cuenta de la protesta magisterial refieren como única fuente la gremial.

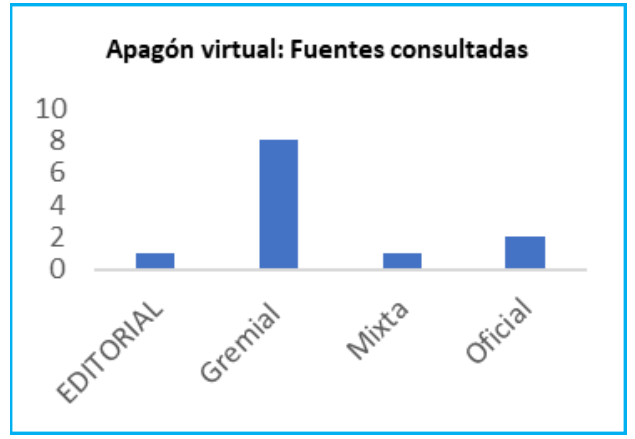

Figura 3

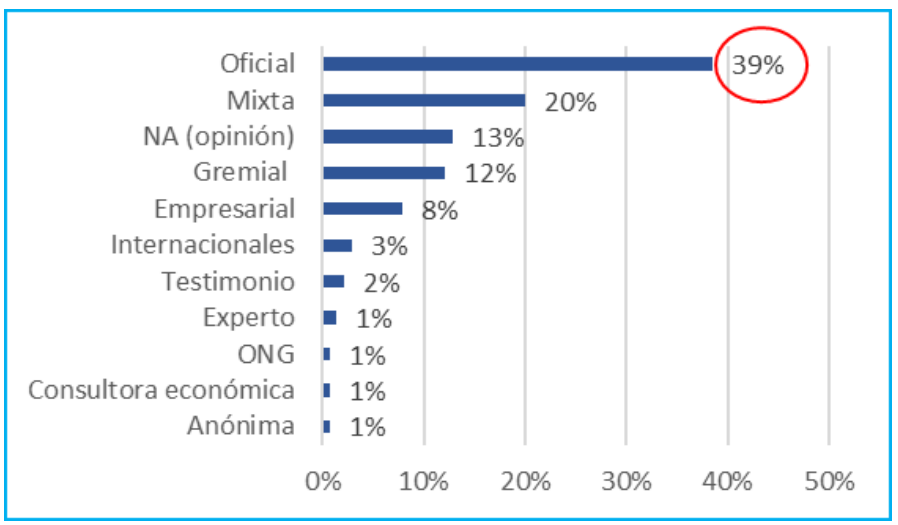

Figura 4 


\section{CONCLUSIONES}

El objetivo de este estudio fue explorar, describir y analizar la cobertura que hicieron los medios de comunicación social de Panamá acerca de la brecha digital durante los seis primeros meses de la pandemia generada por la COVID-19, apoyados en una tradición que se pregunta sobre la calidad de la información que se brinda a las audiencias, como requisito para la existencia de una ciudadanía informada, base de una sociedad democrática.

Aunque con las excepciones de rigor, destaca el abordaje episódico y poco profundo de un tema complejo como la brecha digital, con componentes sociales de fondo que hubiesen requerido la consulta e inclusión de fuentes diversas y un tratamiento más exhaustivo. Desde la elección de los temas susceptibles de ser noticia, pasando por la consulta, análisis y selección de las fuentes, se necesita una cobertura más completa de los asuntos que se tratan y se presentan al público.

Los departamentos de prensa oficiales son oficinas especializadas en la función de posicionar una visión y unos intereses particulares en la opinión pública. Esto lo procuran con mayor determinación los entes gubernamentales, especialmente aquellos excesivamente vigilantes sobre lo que se da a conocer a la población. Durante periodos delicados, como los que demarca una crisis sanitaria, este control se exacerba obstaculizando la labor de los reporteros y, por ende, el adecuado flujo de información a la ciudadanía. En este sentido, aunque - o porque - la labor reporteril se ve obstruida, se requiere un esfuerzo profesional mucho mayor.

En ocasiones también ocurre que la preferencia por la fuente oficial se debe al ejercicio de presiones indebidas por parte de algunos entes del Estado, o a la existencia de intereses cruzados entre actores políticos y propietarios de medios de comunicación. Sin afirmar que esto sea lo ocurrido en Panamá durante la pandemia, en cualquier caso, y cualesquiera sean las razones, el predominio de la fuente única, sea privada o corporativa, es una anomalía periodística que debe combatirse.

Si bien es cierto que el periodismo no es un campo ajeno a la crisis económica desatada por la pandemia - la cual ha ocasionado reducción de personal y de salarios en las salas de redacción de prácticamente todos los medios-, los medios de comunicación, por la delicada labor que cumplen, tienen una responsabilidad social que cumplir. Asimismo, los reporteros, que constituyen uno de los eslabones más frágiles de la cadena de producción de la noticia, deber ser conscientes de la misión tan delicada que desarrollan, como es la de informar a los ciudadanos con calidad.

Gracias a la pandemia, la brecha digital como fenómeno, como asunto de investigación y como tema de comunicación científica, cobró una nueva relevancia. Por delante quedan al menos dos retos: generar más conocimiento contextualizado sobre este problema, y fundamentos profesionales para poder comunicarlo con mayor propiedad. 


\section{Referencias}

[1] van Dijk, Jan. (2020). The Digital Divide. (VitalSource Bookshelf version.).

[2] [Wolf, Mauro. (1987). La investigación en la comunicación de masas. Críticas y Perspectivas. Paidós.

[3] Martini, Stella. (2000). Periodismo, Noticia y Noticiabilidad. Norma.

[4] AEVE PANAMÁ (2020). Comunicado. Coalición Nacional por la Defensa de la Educación Oficial de Panamá

\section{Autorización y Licencia CC}

La autora autoriza a APANAC XVIII a publicar el artículo en las actas de la conferencia en Acceso Abierto (Open Access) en diversos formatos digitales (PDF, HTML, EPUB) e integrarlos en diversas plataformas online como repositorios y bases de datos bajo la licencia CC: Attribution-NonCommercial-ShareAlike 4.0 International (CC BY-NC-SA 4.0) https://creativecommons.org/licenses/by-nc-sa/4.0/.

Ni APANAC XVIII ni los editores son responsables ni del contenido ni de las implicaciones de lo expresado en el artículo. 